(2) Open Access Full Text Article

\title{
Anterior segment optical coherence tomography for demonstrating posterior capsular rent in posterior polar cataract
}

This article was published in the following Dove Press journal:

Clinical Ophthalmology

10 January 2014

Number of times this article has been viewed

\author{
George D Kymionis ${ }^{1,2}$ \\ Vasilios F Diakonis' \\ Dimitrios A Liakopoulos' \\ Konstantinos I Tsoulnaras' \\ Nektarios E Klados' \\ loannis G Pallikaris' \\ Institute of Vision and Optics, \\ Department of Medicine, University \\ of Crete, Heraklion, Crete, Greece; \\ ${ }^{2}$ Bascom Palmer Eye Institute, \\ University of Miami, Miller School of \\ Medicine, Miami, Florida, USA
}

Aims/purpose: To report the preoperative use of anterior segment optical coherence tomography (AS-OCT) for the determination of pre-existing posterior capsule defect in patients with posterior polar cataract.

Methods: Three patients presented with posterior polar cataract and were evaluated preoperatively using AS-OCT, revealing in one patient intact posterior capsule and in the other two a pre-existing posterior capsule defect not detectable by slit-lamp evaluation. All patients underwent phacoemulsification and intraocular lens implantation.

Results: Intraoperatively, AS-OCT findings were confirmed after cataract surgery in all patients. No intra- or late postoperative complications were noted.

Conclusion: AS-OCT could be an additional useful imaging modality in these patients, essential for surgical planning and patient consultation.

Keywords: posterior capsular cataract, phacoemulsification, cataract complications, posterior capsular rupture, anterior segment optical coherence tomography

\section{Introduction}

Posterior polar cataract (PPC) remains a difficult challenge for cataract surgeons because of the high probability of intraoperative nucleus drop. The incidence of posterior capsule rupture during removal of PPC is reported to be as high as $36 \%$, and surgery is of higher risk when there is an associated pre-existing posterior capsule defect. ${ }^{1,2}$ A fundamental differentiation is necessary in PPC patients during their preoperative assessment. It consists of determining whether the posterior capsule is intact or there is a pre-existing posterior capsule defect. The mentioned anatomical difference is of critical importance and may determine both surgical planning and the likelihood of intraoperative complications. ${ }^{1}$

We present three patients with PPC assessed preoperatively using anterior segment optical coherence tomography (AS-OCT) (Visante OCT 3.0; Carl Zeiss Meditec AG, Jena, Germany) for the determination of pre-existing capsule defect; furthermore, we examined the feasibility of this imaging modality as an additional evaluation for improving both surgical planning and patient consultation in cases of PPC.

\section{Case reports}

Three consecutive cases were included in this case series (Table 1). Slit-lamp examination in all patients revealed the presence of PPC with no detectable posterior capsule defect. Ocular history was uneventful for all patients (no trauma), and systemic history revealed increased blood pressure and high cholesterol in cases 1 and 3 .
Correspondence: Vasilios F Diakonis Institute of Vision and Optics, Department of Medicine, University of Crete, 71110 Heraklion, Crete, Greece $\mathrm{Tel}+302810327316$

Fax +302810394653

Email diakonis@gmail.com 
Table I Patient demographics, eye with posterior polar cataract, and visual acuity both preoperative and 3 months postcataract surgery

\begin{tabular}{llllll}
\hline Patient & Age and sex & Eye with PPC & Preoperative UDVA & Postoperative UDVA & Postoperative CDVA \\
\hline 1 & 65 years - male & Left & $20 / 50$ & $20 / 25$ & $20 / 20(-0.50-0.50 \times 90)$ \\
2 & 71 years - male & Right & $20 / 60$ & $20 / 25$ & $20 / 20(-0.75$ sphere $)$ \\
3 & 73 years - male & Left & $20 / 60$ & $20 / 25$ & $20 / 20(-0.75 \times 75)$ \\
\hline
\end{tabular}

Abbreviations: CDVA, corrected distance visual acuity; PPC, posterior capsular cataract; UDVA, uncorrected distance visual acuity.

Postoperatively, all patients demonstrated improvement in both uncorrected and corrected distance visual acuity (Table 1).

\section{Preoperative assessment}

Complete preoperative assessment was performed on all patients, along with AS-OCT with a specific area of interest, the posterior capsule, for the determination of PPC-related defect (Figures 1B and 2B). AS-OCT revealed in respect of case 1 an intact posterior capsule (Figure 1B), whereas cases 2 and 3 demonstrated an area of posterior capsule defect (Figure 2B).

\section{Cataract surgery}

All patients were informed about the type of cataract and the risks involved in surgery; furthermore, they provided a written informed consent for their participation in the study and for the surgical procedure, in accordance with the institutional guidelines and the Declaration of Helsinki. Phacoemulsification was performed under peribulbar anesthesia. This was preferred in case of an unexpected intraoperative complication occurring. Hydrodelineation and gentle viscodissection were performed in all cases. After we removed the endonucleus using low-pressure hydrodelineation, we partially viscodissected (using dispersive viscoelastic) the peripheral epinucleus (by lifting the anterior capsule), avoiding the central part of the capsule (the area of the defect). We removed the epinucleus peripherally toward the center in order to leave a small portion of it at the area of possible defect, which was the last part that we removed. Intraoperatively, an intact posterior capsule was revealed in patient 1 , whereas a posterior capsule defect was found in patients 2 and 3 . Intraoperative findings confirmed the preoperative AS-OCT results. Anterior vitrectomy was performed in the patients with posterior capsule defect due to posterior capsule rupture extension. A three-piece intraocular lens was placed into the sulcus in these patients.

No intra- or postoperative complications were noticed in the three cases, such as intraocular lens dislocation or development of cystoid macular edema.

\section{Discussion}

The aim of the surgical approach in cases with PPC is to minimize turbulence and pressure in the anterior chamber and the capsular bag, thereby preventing iatrogenic posterior capsule rupture or extension of a pre-existing posterior capsule defect. ${ }^{3-6}$ To achieve this, slow-motion phacoemulsification and gentle hydrodelineation along with gentle viscodissection or hydrodissection have been proposed. ${ }^{1,3}$ Furthermore, Vasavada and $\mathrm{Raj}^{7}$ propose a technique to achieve precise hydrodelineation, which they name "insideout delineation." This approach eliminates the possibility of fluid being injected inadvertently into the subcapsular plane, leading to unwarranted hydrodissection, and leaving
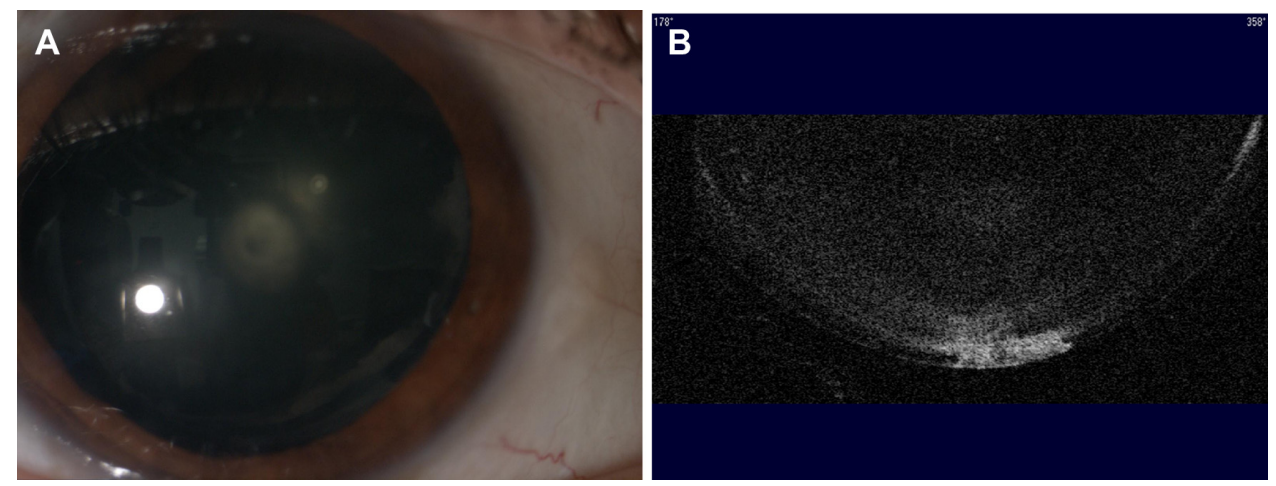

Figure I (A) Slit-lamp image demonstrating a patient with posterior polar cataract. (B) Anterior segment optical coherence tomography, focusing at the posterior part of the crystalline lens, demonstrating an intact posterior capsule (images of case I). 

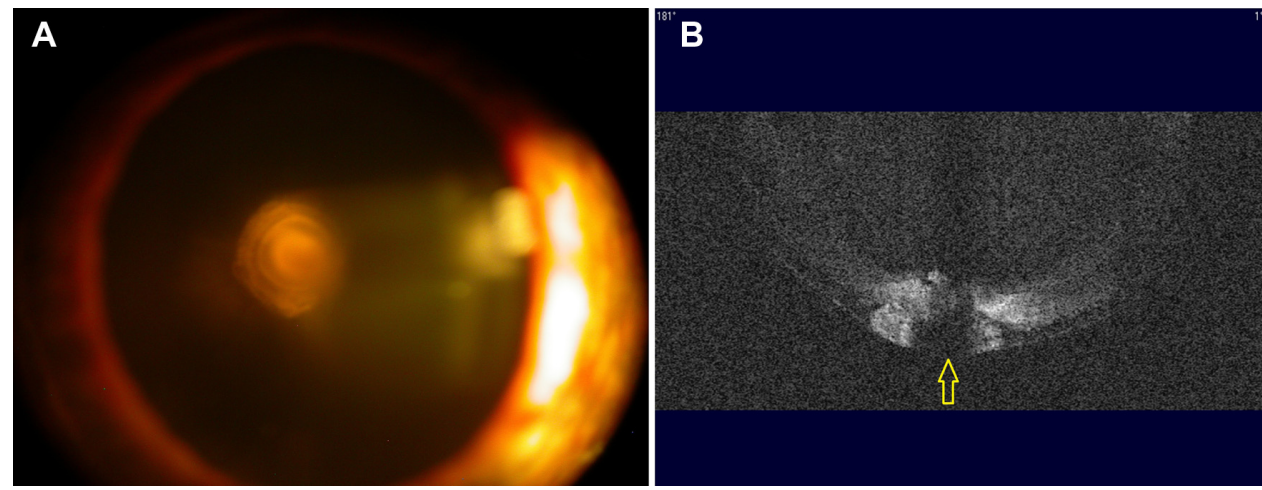

Figure 2 (A) Slit-lamp image demonstrating a patient with posterior polar cataract. (B) Anterior segment optical coherence tomography, focusing at the posterior part of the crystalline lens, demonstrating a discontinuation defect at the posterior capsule (yellow arrow) (images of case 2).

an intact cortical shell, which prevents the extension of a posterior capsular rent.

In our case studies, we utilized AS-OCT, an imaging modality that overcomes the drawbacks of slit-lamp examination. We were able to detect a posterior capsule rupture in cases 2 and 3 , and an intact capsule in case 1 . A possible limitation of this imaging technology could be the inability of detection of a "true" capsular defect, as the optical density of the overlaying lens opacity could create false positive outcomes. Nevertheless, in our cases, surgery confirmed the AS-OCT findings. All cases achieved satisfactory postoperative visual acuity.

Iatrogenic posterior capsule rupture may also occur without its pre-existence. This mainly occurs due to the crystalline lens adhesions on the posterior capsule and the surgical maneuvering during cataract extraction. Nevertheless, posterior capsule pre-existing defects increase significantly the possibility of nucleus drop and the need for posterior segment interventions.

\section{Conclusion}

It seems that AS-OCT may be utilized as an additional modality for the identification of pre-existing capsule rupture in patients with PPC. Even though this is only a three-patient series and final conclusions may not be drawn on the impact AS-OCT may have on the management of PPC, it seems that it is feasible to detect a discontinuation of the posterior capsule using this technology.

\section{Disclosure}

No author has financial or proprietary interest in any product, method, or material used in this study.

\section{References}

1. Vasavada AR, Raj SM, Vasavada V, Shrivastav S. Surgical approaches to posterior polar cataract: a review. Eye. 2012;26:761-770.

2. Ghosh YK, Kirkby GR. Posterior polar cataract surgery: a posterior segment approach. Eye. 2008;22:844-848.

3. Singh K, Mittal V, Kaur H. Oval capsulorhexis for phacoemulsification in posterior cataract with preexisting posterior capsule rupture. $J$ Cataract Refract Surg. 2011;37:1183-1188.

4. Fine IH, Packer M, Hoffman RS. Management of posterior polar cataract. $J$ Cataract Refract Surg. 2003;29:16-19.

5. Siatiri H, Moghimi S. Posterior polar cataract: minimizing risk of posterior capsule rupture. Eye. 2006;20:814-816.

6. Hayashi K, Hayashi H, Nakao F, Hayashi F. Outcomes of surgery for posterior polar cataract. $J$ Cataract Refract Surg. 2003;29:45-49.

7. Vasavada AR, Raj AM. Inside-out delineation. $J$ Cataract Refract Surg. 2004;30(6):1167-1169
Clinical Ophthalmology

\section{Publish your work in this journal}

Clinical Ophthalmology is an international, peer-reviewed journa covering all subspecialties within ophthalmology. Key topics include: Optometry; Visual science; Pharmacology and drug therapy in eye diseases; Basic Sciences; Primary and Secondary eye care; Patient Safety and Quality of Care Improvements. This journal is indexed on

\section{Dovepress}

PubMed Central and CAS, and is the official journal of The Society of Clinical Ophthalmology (SCO). The manuscript management system is completely online and includes a very quick and fair peer-review system, which is all easy to use. Visit http://www.dovepress.com/ testimonials.php to read real quotes from published authors. 